\title{
Efeitos de um programa educacional de autocuidado de coluna em idosos com dor lombar crônica: um estudo quasi- experimental
}

\author{
Effects of an educational self-care program of spine in elderly patients \\ with chronic low back pain: a quasi-experimental study
}

Julia Catarina Sebba Rios ${ }^{1 *}$, Tailce Kaley Moura Leite ${ }^{1}$, Márcio de Moura Pereira², Fabiany Calixto Sousa ${ }^{1}$, Marisete Peralta Safons ${ }^{1}$

ARTIGO ORIGINAL | ORIGINAL ARTICLE

\begin{abstract}
O estudo teve por objetivo verificar os efeitos de um programa educacional de autocuidado de coluna, no controle da dor e na incapacidade em idosos ativos com dor lombar crônica (DL) não específica. Os idosos foram divididos em grupo experimental (GE $n=17)$ e controle $(\mathrm{GC} n=13)$. A intervenção consistiu na entrega de uma cartilha educativa e um workshop. Foram variáveis dependentes: 1. intensidade da dor (EVN), 2. capacidade funcional relacionada à DL (RMQ), 3. quantidade de pontos de dor, 4. uso de medicação para DL e 5. frequência de uso de medicação para DL. Houve avaliação pré e após 1 mês da primeira avaliação. Na avaliação intragrupo foi observada no GE diminuição significativa de todos os parâmetros avaliados (todos $\mathrm{p}<0,05$ ) fato não observado no GC. Quanto à análise entre os grupos houve melhora significativa com tamanhos de efeitos moderados a grandes no GE para EVN $(p=0.003$, TE=0.53), no RMQ $(p=0.037$, TE $=0.38)$ e nos pontos de dor $(p=0.006$, $\mathrm{TE}=0.49$ ). Os resultados demonstraram que a cartilha pode ser uma ferramenta útil, capaz de gerar independência no cuidado e tratamento da DL crônica em idosos ativos.
\end{abstract}

Palavras-chave: Lombalgia, idoso, autocuidado, educação

ABSTRACT

The purpose of this paper was to investigate the effects of an educational self-care program of spine in the control of pain and disability in actives older adults with chronic non-specific low back pain (LBP). They were divided in an experimental group (EG $n=17)$ and a control group $(C G n=13)$. The intervention consisted of delivering an educational booklet and also attending a workshop. Dependent variables were: 1. pain intensity (EVN), 2. functional capacity related to LBP (RMQ), 3. amount of pain points, 4 . medication for LBP, 5 . frequency of medication for LBP. There was evaluation pre and after 1 month of the first evaluation. The intragroup evaluation was observed in EG significant reduction of all parameters (all p < 0.05) was not observed in CG. Regarding the analysis between groups showed significant improvement with moderate to large effect sizes in EG to EVN ( $p=0.003, T E=0.53)$, RMQ $(p=0.037, T E=0.38)$ and pain points $(p=0.006, T E=0.49)$. The results highlight the booklet as a useful tool, capable of generating independence in the self-care for active older adults with chronic LBP. Keywords: Low back pain, aged, self-care, education

Artigo recebido a 29.10.2013; Aceite 05.06.2014

${ }^{1}$ Universidade de Brasília, UnB, Brasil

${ }^{2}$ Universidade Católica de Brasília, UCB, Brasil

* Autor correspondente: SQS 414 bloco E apt. 104, Brasília DF CEP:70297 050, Brasil;

E-mail: jcatarina@gmail.com 


\section{INTRODUÇÃO}

A dor lombar (DL) não específica é um grande problema de saúde pública mundial (Balagué, Mannion, Pellisé, \& Cedraschi, 2012) e é considerada crónica quando persiste por mais de 3 meses (Bogduk, 2004). Esse problema complexo é um dos mais prevalentes na população idosa (Dionne, Dunn, \& Croft, 2006; Lima et al., 2009; van den Bussche et al., 2011) e está associado a consequências adversas, incluindo incapacidade funcional, alteração psicossocial, aumento do uso de recursos de saúde, além de apresentar influências negativas na qualidade de vida, na saúde e independência dos idosos (Hicks, Gaines, Shardell, \& Simonsick, 2008).

Considerando a alta prevalência da DL associada à dificuldade de tratar adequadamente essa condição, várias diretrizes de prática clínica recentes têm tentado resumir as evidências científicas da literatura (Airaksinen et al., 2006; Brazil et al., 2004; Dagenais, Tricco, \& Haldeman, 2010). Dentre as intervenções recomendadas pela literatura estão educação, exercícios e o autocuidado (Arnau et al., 2005; Balagué et al., 2012; Dagenais et al., 2010; May, 2010).

$\mathrm{O}$ autocuidado se refere a habilidade do indivíduo em gerenciar os sintomas, o tratamento, suas consequências físicas e psicológicas e mudanças no estilo de vida que são inerentes a viver com uma condição crônica (Barlow, Wright, Sheasby, Turner, \& Hainsworth, 2002). Ele serve para modificar os comportamentos de saúde, a condição da saúde, além de encorajar e ensinar os pacientes a identificar e solucionar problemas, definir metas e planejar ações (May, 2010). Ademais, o autocuidado pode ser uma alternativa economicamente atraente, dado seu potencial, na redução de dependência do sistema de saúde, pois nesse modelo os pacientes estão ativamente envolvidos no cuidado de sua DL (Barlow et al., 2002).

A diretriz brasileira para tratamento de lombalgias e lombociatalgias recomenda e aponta que a educação e o esclarecimento dos pacientes são fundamentais para a reabilitação (Brazil et al., 2004). Segundo revisão feita por May (2010), exercício baseado em conselhos e educação deve ser a chave para as estratégias de autocuidado da DL.

No entanto, apesar de ser endossado pela maioria dos guias clínicos (Balagué et al., 2012; Dagenais et al., 2010; May, 2010), uma revisão sistemática recente com meta-análise, que teve por objetivo verificar a efetividade do autocuidado da DL não-específica, demonstrou evidência de qualidade moderada, com pequenos efeitos na melhora da dor e incapacidade (Oliveira et al., 2012). Outra revisão sistemática, feita pela Cochrane Collaboration (Engers et al., 2008), verificou que educação do paciente individual é tão efetiva quanto outras intervenções em longo prazo para dor e melhora geral na DL aguda e subaguda. Porém, para DL crônica, o valor da educação ainda não está estabelecido, pois há uma escassez de trabalhos. Nesta revisão de Engers et al. (2008) não foram incluídos estudos com educação em grupo e nem que continham exercícios. Ambas as revisões incluíram pesquisas em indivíduos com mais de 18 anos e concordam quanto à necessidade de mais pesquisas para esse desafiante problema, que é a DL crônica não específica.

Dentre os estudos que investigaram efeitos associados de programas educacionais e de autocuidado relacionados à DL aguda e cróni$\mathrm{ca}$, resultados significativos foram demonstrados no que se refere ao controle da dor e da incapacidade, incremento da atividade física, diminuição do medo, redução da ansiedade/depressão, aumento da qualidade de vida e diminuição de reincidências e frequência dos sintomas (Albaladejo, Kovacs, Royuela, del Pino, \& Zamora, 2010; Damush et al., 2003; Heymans, van Tulder, Esmail, Bombardier, \& Koes, 2004; Meng et al., 2011; Udermann et al., 2004). Porém, poucos autores investigaram seus efeitos na DL crônica da população idosa (Haas et al., 2005; Kovacs et al., 2007), encontrando resultados positivos e significativos apenas na incapacidade funcional relacionada à 
DL dentre as várias variáveis estudadas como: intensidade de dor, saúde geral, qualidade de vida, entre outras.

Portanto, o valor de programas educacionais de autocuidado da DL crônica no contexto da população idosa é incerto, e verificar os efeitos de um programa educacional de autocuidado de coluna pode servir de base para aperfeiçoar as abordagens de tratamento existentes visando à independência do paciente, a melhora funcional e clínica, assim como os cuidados e prevenção da piora desses sintomas dolorosos que podem conduzir o idoso a perdas progressivas da qualidade de vida e tendem a persistir por mais tempo nessa faixa etária (Hicks et al., 2008; Leboeuf-Yde, Nielsen, Kyvik, Fejer, \& Hartvigsen, 2009).

Diante disso, o objetivo do presente estudo foi verificar os efeitos de um programa educacional de autocuidado de coluna no controle da dor e na incapacidade em idosos ativos com dor lombar crônica não específica.

\section{MÉTODO}

O estudo se caracteriza como ensaio clínico controlado e não aleatório e foi devidamente aprovado pelo Comitê de Ética e Pesquisa da Faculdade de Medicina da Universidade de Brasília (UnB), número CEP-FM 045/2011.

\section{Amostra}

A amostra foi de conveniência e selecionada em um programa de exercícios físicos oferecido pelo Grupo de Estudos e Pesquisas sobre Atividade Física para Idosos da Universidade de Brasília (GEPAFI - UnB), em 2011. Todos os idosos $(n=193)$ que frequentam as atividades desse programa têm autorização médica para praticar exercício físico e estão vinculados a no mínimo uma modalidade oferecida pelo programa (musculação, yoga, dança, pilates solo e corrida).

Os participantes do estudo foram préselecionados considerando os relatos de DL descritos na ficha de avaliação padrão do programa. A partir da pré seleção $(n=88)$, foi aplicado um questionário de triagem que conside- rou os seguintes critérios de inclusão: (1) ter idade entre 60 e 75 anos; (2) apresentar quadro de dor lombar local ou referida acima do joelho há mais de três meses; (3) saber ler e escrever. Foram excluídos os sujeitos que preencheram algum dos seguintes critérios: (1) ter sintomas de dor abaixo do joelho; (2) estar em tratamento específico para DL; (3) ter história de cirurgia na coluna ou de doença inflamatória sistêmica; (4) ter sofrido acidente ou trauma nos últimos três meses; (5) ter anestesia na região interna da coxa ou perineal; (6) apresentar comprometimento grave de visão ou audição; (7) ser portador de comorbidade grave; (8) vir apresentando perda de peso sem razão. Os critérios de exclusão serviram para excluir indícios de causas específicas de DL (bandeiras vermelhas) e, dessa forma classificar a DL como não específica. Pelos critérios acima foram excluídos 28 sujeitos, outros idosos pré selecionados $(n=25)$ não estavam frequentando momentaneamente as atividades do grupo por diversos motivos como doença pessoal ou famíliar e viagens e não puderam ser triados e 5 idosos não quiseram participar.

Em seguida, os idosos $(n=30)$ foram devidamente informados sobre os objetivos, procedimentos e possíveis implicações da participação na pesquisa e convidados a assinar o termo de consentimento livre e esclarecido (TCLE).

\section{Instrumentos}

Foi aplicada a Escala Visual Numérica (EVN) para avaliação da intensidade da dor, onde os idosos foram instruídos a dar uma pontuação média para a dor numa escala visual de 0 (nenhuma dor) a 10 (dor insuportável) (Jensen, Karoly, \& Braver, 1986). E para a avaliação da incapacidade relacionada com a dor lombar, o Original and Brazilian-Portuguese version of the Roland-Morris Questionnaire (RMQ). Amplamente utilizado em pesquisas, o RMQ é um instrumento rápido e fácil de administrar, composto por uma lista de 24 frases que abordam o desempenho funcional em relação à dor nas costas, frases essas que descrevem situações diárias que o indivíduo tem difi- 
culdade de realizar por causa das dores nas costas. É atribuído um ponto a cada frase e, quanto maior a pontuação final (somatória), maior é a incapacidade do indivíduo (Nusbaum, Natour, Ferraz, \& Goldenberg, 2001). Um número substancial de artigos foi publicado sobre as propriedades psicométricas, a validade e a confiabilidade desse questionário (Costa, Maher, Latimer, et al., 2007; Costa, Maher, \& Latimer, 2007).

Para avaliação complementar da dor foi utilizado um diagrama do corpo, no qual os sujeitos foram orientados a pintar os pontos de dor sentidos na última semana (centro da coluna, nádega, etc.). E um questionário formulado pelos pesquisadores que aborda a utilização de medicação para ajudar a controlar a dor lombar, quanto ao uso (sim ou não) e à frequência de utilização no último mês (nunca; raramente; 1 a 2 vezes na semana; 3 a 4 vezes na semana ou diariamente).

Foi aplicado também o Questionário Internacional de Atividade Física (IPAQ) - Versão Curta (Matsudo et al., 2001), que classifica o indivíduo em muito ativo, ativo, irregularmente ativo A e B e sedentário, e que foi utilizado para a caracterização do nível de atividade física da amostra.

Para um controle qualitativo mais efetivo na avaliação pós intervenção foi utilizado um questionário idealizado pelos pesquisadores para auxiliar na interpretação da adesão ao programa de autocuidado proposto, com questões referentes à leitura da cartilha, à realização dos exercícios propostos e à utilização do rolo lombar na posição sentada.

\section{Procedimentos}

A distribuição nos grupos de intervenção e controle foi não aleatória. Os grupos foram formados no dia da primeira avaliação, considerando a disponibilidade dos idosos para participar da intervenção nos dias previamente programados. O delineamento da pesquisa está exposto na figura 1 .

O grupo experimental recebeu um programa educacional de autocuidado com as seguin- tes estratégias: (1) uma Cartilha de Autocuidado de Coluna, adaptada e baseada no livro "Trate você mesmo sua Coluna", de Robin McKenzie (2007), livro apontado como ferramenta capaz de modificar comportamento e reduzir significativamente a DL crônica em pacientes adultos (Udermann et al., 2004), e (2) um workshop.

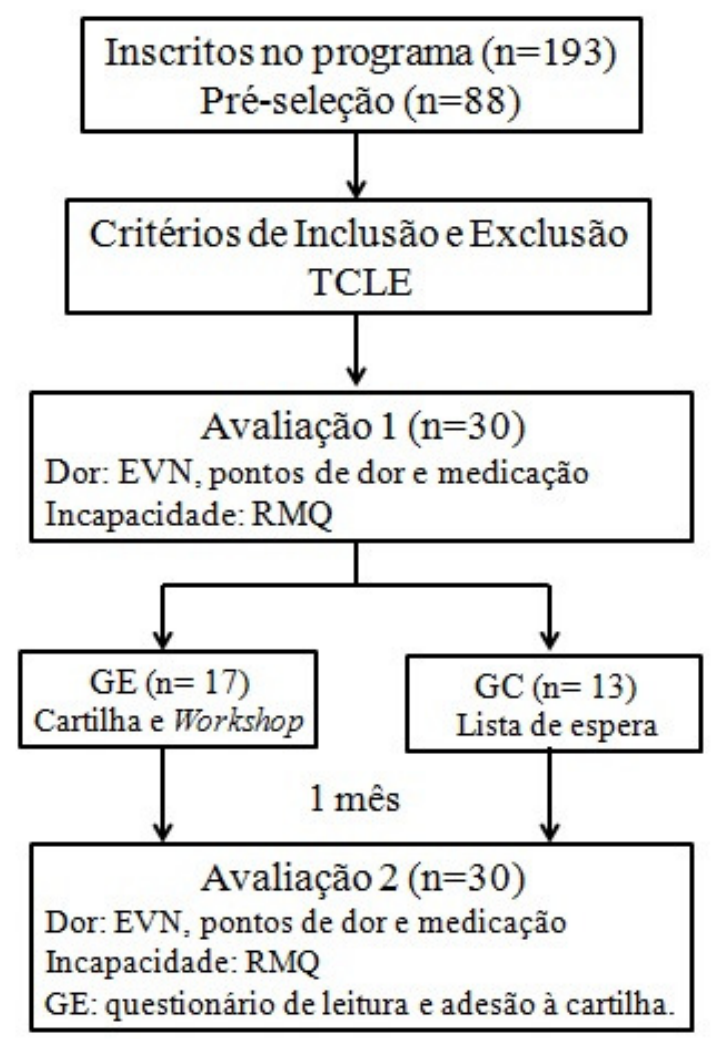

Figura 1. Delineamento da pesquisa

TCLE $=$ Termo de consentimento livre e esclarecido; IPAQ = Questionário Internacional de Atividade Física; EVA = Escala Analógica Visual; RMQ = Rolland Morris Questionnaire; GE = grupo experimental; GC = grupo controle

A intervenção consistiu de um encontro com o GE no qual houve a entrega da Cartilha de Autocuidado de Coluna, ilustrativa e educativa, com informações básicas sobre a coluna vertebral, a importância da lordose, a origem mecânica da dor lombar, autocuidado postural em posições variadas e interpretação de modificações na localização e intensidade da dor. O componente de autotratamento da cartilha continha instruções claras e ilustradas sobre 
(1) a manutenção da lordose lombar em variadas posições da vida diária, (2) o uso de um suporte lombar quando sentado e (3) sobre exercícios de extensão da coluna lombar (deitado em prono, prono extensão, extensão repetida deitado ou extensão repetida em pé). Os exercícios são indicados com o objetivo de reposicionar as articulações, diminuir, abolir ou centralizar a dor e melhorar a movimentação da coluna (sugestão de frequência: 5 séries ao dia, 7 a 10 repetições por série).

Além da cartilha, o GE recebeu um workshop de 30 minutos de duração que teve como objetivos: reforçar a importância da leitura da cartilha, ensinar na prática como fazer um rolo lombar adaptado (uso de toalha) e permitir a vivência dos exercícios propostos com supervisão e correção. O grupo controle ficou em lista de espera e recebeu a mesma intervenção após a finalização do estudo.

Os grupos foram reavaliados um mês após a primeira avaliação, e além dos questionários de dor, medicação e incapacidade, foram coletados dados qualitativos acerca da adesão ao programa de autocuidado, leitura e interpretação da cartilha. As avaliações das variáveis dependentes foram realizadas por colaboradores treinados e cegos para os grupos.

\section{Análise Estatística}

Foi realizada análise exploratória dos dados, e as variáveis foram apresentadas em média e desvio padrão. A normalidade dos dados foi testada pelo teste Shapiro-Wilk. Aplicaram-se os Testes $t$ independente, U de Mann-Whitney e Qui-quadrado para comparar os dados no momento inicial do estudo. Como não foi observada a normalidade nas variáveis RMQ, pontos de dor e frequência de medicação, foi utilizado o teste não paramétrico de Wilcoxon para comparação intragrupos de pré e pósteste, já para a EVN, aplicou-se o teste $\mathrm{t}$ pareado. Para comparação da frequência observada e esperada de pessoas em uso de medicação para DL, foi utilizado o teste de Qui-quadrado. Para comparação do delta (variação entre os testes pré e pós-intervenção) dos grupos (controle e intervenção), foi utilizado o teste de Mann-Whitney nas variáveis RMQ pontos de dor e frequência de medicação e $\mathrm{o}$ teste $\mathrm{t}$ independente para a EVN. Em todas as análises em que se verificou diferença significativa da intervenção sobre alguma variável, o tamanho deste efeito (TE) foi testado, adotando-se o critério de Cohen. As análises estatísticas foram desenvolvidas no programa SPSS 17.0, adotando-se como nível de significância $\mathrm{p} \leq 0.05$.

Tabela 1

Caracterização Amostral

\begin{tabular}{|c|c|c|c|}
\hline Características & $\mathrm{GE}(\mathrm{n}=17)$ & $\mathrm{GC}(\mathrm{n}=13)$ & $\mathrm{p}$ \\
\hline Idade $(\bar{x} \pm \mathrm{DP})$ & $66.59 \pm 5.08$ & $65.64 \pm 4.30$ & $0.545^{\mathrm{a}}$ \\
\hline Sexo feminino $\mathrm{n}(\%)$ & $14(82.4 \%)$ & $12(92.3 \%)$ & \\
\hline Tempo de dor $(\bar{x} \pm \mathrm{DP})$ & $13.2 \pm 31.8$ & $8.6 \pm 26.4$ & $0.384^{c}$ \\
\hline Escolaridade (\%) & & & $0.680^{\mathrm{d}}$ \\
\hline Básico & $1(5.9 \%)$ & $1(7.7 \%)$ & \\
\hline Fundamental & $1(5.9 \%)$ & $0(0 \%)$ & \\
\hline Médio & $2(11.8 \%)$ & $1(7.7 \%)$ & \\
\hline Superior & $13(76.5 \%)$ & $10(84.6 \%)$ & \\
\hline IPAQ (curta) n (\%) & & & $0.209^{c}$ \\
\hline Muito Ativo & $4(23.5 \%)$ & $0(0 \%)$ & \\
\hline Ativo & $9(52.9 \%)$ & $8(61.5 \%)$ & \\
\hline Irregularmente $\mathrm{A}$ & $1(5.9 \%)$ & $4(30.8 \%)$ & \\
\hline Irregularmente B & $3(17.6 \%)$ & $1(7.7 \%)$ & \\
\hline
\end{tabular}




\section{RESULTADOS}

A caracterização amostral dos sujeitos que preencheram os critérios para inclusão na pesquisa e assinaram o TCLE $(n=30)$ está exposta na Tabela 1, e demonstra que não foram observadas diferenças na linha de base.

Após um mês da intervenção, foram verificados efeitos positivos significativos no grupo experimental de autocuidado de coluna, efeitos esses não observados no grupo controle - Tabelas 2 a 4.

No grupo experimental (GE), foi observada diminuição significativa com grandes tamanhos de efeito em todas as variáveis dependentes: 1 intensidade da dor - EVN $(\mathrm{p}<0.003$ e TE $=$ 0.69), 2 - incapacidade relacionada à dor lombar - RMQ ( $<0.001$, TE $=0.75), 3$ - número de pontos de dor $(\mathrm{p}<0.001$, TE $=0.73), 4$ número de pessoas em uso de medicação para controle da DL $(\mathrm{p}=0.002$, TE $=0.54)$ e 5 frequência de utilização da medicação ( $\mathrm{p}<$ 0.031 , TE $=0.54$ ). No grupo controle não foi percebida nenhuma alteração significativa das variáveis estudadas (Tabelas 2 e 3).

Tabela 2

Comparação pré X pós teste

\begin{tabular}{ccccccc}
\hline & \multicolumn{3}{c}{ Grupo Experimental $(\mathrm{n}=17)$} & \multicolumn{2}{c}{ Grupo Controle $(\mathrm{n}=13)$} \\
& Pré-teste & Pós-teste & $\mathrm{p}$ & Pré-teste & Pós-teste & $\mathrm{p}$ \\
\cline { 2 - 7 } EVN $^{\mathrm{a}}$ & $5.65 \pm 1.41$ & $2.71 \pm 2.78$ & $0.002^{*}$ & $5.77 \pm 2.32$ & $6.38 \pm 2.18$ & 0.373 \\
$\mathrm{RMQ}^{\mathrm{b}}$ & $5.88 \pm 4.78$ & $3.41 \pm 3.00$ & $<0.001^{*}$ & $8.31 \pm 5.65$ & $7.69 \pm 5.60$ & 0.305 \\
Pontos de Dor $^{\mathrm{b}}$ & $2.00 \pm 1.37$ & $0.65 \pm 0.70$ & $0.001^{*}$ & $1.69 \pm 0.86$ & $1.31 \pm 0.48$ & 0.188 \\
Freq. Medicação $^{\mathrm{b}}$ & $0.94 \pm 1.44$ & $0.18 \pm 0.53$ & $0.031^{*}$ & $0.92 \pm 1.32$ & $0.77 \pm 1.17$ & 0.750 \\
\hline
\end{tabular}

${ }^{*}$ Estatisticamente significativo. EVN = Escala Visual Numérica; RMQ = Rolland Morris Questionnaire; ${ }^{2}$ Teste t independente; ${ }^{\mathrm{b}}$ Teste de Wilcoxon.

Foi explorado também o percentual de variação (delta relativo) do pós-teste para o préteste entre as variáveis mensuradas, observando-se de forma clara as diferenças entre os grupos controle e experimental em resposta ao programa educativo aplicado, comprovadas estatisticamente pela comparação entre esses grupos e observado os tamanhos de efeito de moderados a grandes nas variáveis EVN, RMQ e pontos de dor (Tabela 4).

Tabela 3

Comparação pré e pós teste (uso de medicação)

\begin{tabular}{ccccc}
\hline & \multicolumn{2}{c}{ Grupo Experimental $(\mathbf{n}=17)$} & \multicolumn{2}{c}{ Grupo Controle $(\mathbf{n}=13)$} \\
Medicação para DL & Pré-teste & Pós-teste & Pré-teste & Pós-teste \\
\hline Não & $10(58.82 \%)$ & $15(88.24 \%)$ & $7(53.85 \%)$ & $8(61.54 \%)$ \\
Sim & $7(41.18 \%)$ & $2(11.76 \%)$ & $6(46.15 \%)$ & $5(38.46 \%)$ \\
\hline
\end{tabular}

${ }^{*}$ Estatisticamente significativo; ${ }^{\mathrm{d}}$ Teste qui-quadrado.

A partir da análise qualitativa dos dados pós-teste do GE, foi observado que $88.2 \%$ dos sujeitos leram ao menos 1 vez a cartilha de autocuidado de coluna; $58.82 \%$ relataram fazer os exercícios descritos no mínimo 3 vezes ao dia e $17.65 \%$ uma vez ao dia (abaixo do recomendado) e a maioria, $58.82 \%$, utiliza o rolo lombar na posição sentada sempre ou quase sempre. 
Tabela 4

Comparação da variação relativa (\% delta) entre os grupos

\begin{tabular}{|c|c|c|c|c|}
\hline \multicolumn{5}{|c|}{ Variação Relativa } \\
\hline & GE & GC & $\mathrm{p}$ & TE \\
\hline EVN - Intensidade & $-49.48 \%$ & $+55.94 \%$ & $0.003^{* a}$ & 0.53 \\
\hline RMQ - Incapacidade & $-33.07 \%$ & $-01.05 \%$ & $0.037^{* c}$ & 0.38 \\
\hline Pontos de Dor & $-57.25 \%$ & $-10.25 \%$ & $0.006^{* c}$ & 0.49 \\
\hline Medicação & $-29.41 \%$ & $-07.69 \%$ & $0.427^{c}$ & 0.16 \\
\hline Freq. Medicação & $-31.37 \%$ & $-01.92 \%$ & $0.528^{c}$ & 0.14 \\
\hline
\end{tabular}

*Estatisticamente significativo; TE = tamanho de efeito; EVN = Escala Visual Numérica de dor; RMQ = Rolland Morris Questionnaire; ; ${ }^{\mathrm{T} T e s t e} \mathrm{t}$ independente, ${ }^{\mathrm{C}}$ Teste U de Mann-Whitney.

\section{DISCUSSÃO}

Nesta pesquisa de intervenção única e período curto de acompanhamento (1 mês), foram constatadas melhorias significativas com grandes tamanhos de efeito em todas as variáveis dependentes (EVN, RMQ pontos de dor, número de pessoas em uso de medicação para DL e da frequência de utilização dessa medicação) na avaliação intragrupo. Já na análise entre os grupos, houve melhora significativa com tamanhos de efeitos moderados a grandes no GE para EVN, RMQ e nos pontos de dor, após o desenvolvimento do programa educacional de autocuidado de coluna, em idosos ativos com dor lombar crônica não específica.

Esses resultados do GE também se mostram clinicamente importantes, pois atingem uma melhora maior que $20 \%$ tanto para intensidade da dor quanto para incapacidade relacionada à DL (Bombardier, Hayden, \& Beaton, 2001). E ainda revelam que um instrumento simples pode educar e encorajar o paciente a ter independência e responsabilidade no controle da sua DL crônica.

Embora não tenha sido observada diminuição significativa no delta relativo para utilização de medicação para ajudar no controle da DL, observou-se uma redução percentual maior no grupo experimental (- 29.41\%) relativamente ao grupo de controle $(-7.69 \%)$, o que aponta uma tendência ao menor uso de medicamento pelo GE. Além disso, foi observada ainda uma redução estatisticamente significativa do número de pessoas em uso de medicação para DL dentre aqueles que passaram pelo programa educacional de autocuidado.

Esses achados vêm corroborar com os de Udermann et al. (2004), que se propuseram a verificar se a leitura do livro "Trate você mesmo sua coluna" (McKenzie, 2007) obra na qual se baseou o presente estudo, poderia modificar o comportamento e reduzir a dor lombar crônica em pacientes adultos $(n=62)$. Em 9 meses de acompanhamento, os resultados desses autores mostraram que essa conduta apresentou eficácia clínica considerável na diminuição da dor ( $\mathrm{p}<0.03)$, na redução de número de episódios de dor ( $p<0.0001)$, em uma menor utilização dos serviços de saúde e no benefício percebido pelos sujeitos $(p<0.04)$, sendo que em 18 meses os ganhos foram mantidos ou apresentaram melhora ainda maior. E sugerem que a cartilha adaptada proposta neste estudo pode ser uma opção mais barata e possivelmente mais aceita no Brasil, por ser resumida e mais simples. No entanto, futuras investigações devem ser realizadas a fim de elucidar melhor essa inferência.

O autocuidado, incluindo a educação acerca de comportamento saudável, é visto como um ingrediente importante no tratamento apropriado e compreensivo de saúde e vem sendo recomendado pela maioria dos guias clínicos para tratamento da dor lombar (Dagenais et al., 2010). As crenças pessoais têm sido associadas à adoção de comportamentos saudáveis (Clark, 1996) que são essenciais para o sucesso terapêutico e para melhora das condições fun- 
cionais do envelhecimento (Vita, Terry, Hubert, \& Fries, 1998).

E, apesar de achados de uma revisão recente apontarem que a evidência de eficácia de autocuidado para DL não é clara, apresentando evidência de qualidade moderada com pequenos efeitos sobre dor e incapacidade (Oliveira et al., 2012), os resultados do presente estudo e de outras pesquisas (Heymans et al., 2004) observaram diminuição da dor local/referida e da incapacidade. Outros estudos indicam redução de outras variáveis como o medo, a ansiedade/depressão, as reincidências e atitudes catastróficas, além do incremento da atividade física, do aumento da qualidade de vida (Albaladejo et al., 2010; Damush et al., 2003; Meng et al., 2011; Udermann et al., 2004).

Porém, no universo da população idosa, apenas dois estudos envolvendo educação e autocuidado na DL foram encontrados, e apresentaram métodos diferentes do presente estudo. Kovacs et al. (2007) realizaram estudo com idosos institucionalizados $(\mathrm{n}=661)$ e idade média de 81,2 anos com e sem DL. Os idosos foram randomizados em 3 grupos educacionais, todos receberam uma palestra resumo de 20 minutos, sendo o G1 grupo de educação ativa mais um livro de coluna; o G2 grupo de higiene postural mais um guia de coluna e o G3 grupo controle (folheto cardiovascular). Observaram que, a entrega do livro de coluna com foco na educação ativa com estímulo a mudanças de atitudes e comportamentos (G1) melhora estatisticamente a incapacidade (RMQ) em um acompanhamento de 6 meses, mas é clinicamente irrelevante. Além desse, o estudo randomizado e controlado de Haas et al. (2005) que teve o objetivo de avaliar a efetividade de um programa de autogerenciamento (6 semanas, 2 horas e meia por semana) para doença crônica de Stanford's usado para DL crônica de origem mecânica em idosos americanos da comunidade $(\mathrm{n}=109)$, idade média de 77,2 anos, observou que não há vantagem para melhora da dor, saúde geral, autoeficácia e atitudes de autocuidado. No entanto, houve benefício sugerido para saúde emocional, incapacidade funcional e dias de incapacidade.

As diretrizes de prática clínica apontam que exercícios supervisionados são eficazes na redução de dor e melhora funcional de indivíduos com DL crônica não específica (Airaksinen et al., 2006; van Middelkoop et al., 2010). E, os dados encontrados no presente estudo e no de Kovacs et al. (2007) refletem que exercícios autogeridos também podem ser eficazes, e assim apresentar melhor custobenefício na população idosa.

Algumas estratégias de autotratamento suportadas pela literatura foram utilizadas nesse curto programa de autocuidado de coluna, pois a cartilha e o workshop envolviam a educação e capacitação do paciente para o monitoramento adequado dos sinais e sintomas para o gerenciamento da sua dor lombar (Balagué et al., 2012). Um dos aspectos que o programa enfatizou foi a importância da lordose lombar em posições variadas, e especialmente, na postura sentada, com sugestão de uso de um suporte (rolo lombar). Essa recomendação é sustentada por algumas pesquisas que apontam a postura lordótica ao sentar como sendo ideal e ótima para saúde da coluna, pois diminui a pressão intradiscal e a tensão nos ligamentos, melhora a distribuição de carga na coluna entre discos e articulações zigoapofisárias, reduzindo assim a degeneração discal e protegendo seus tecidos (Harrison, Harrison, Croft, Harrison, \& Troyanovich, 1999; Pynt, Higgs, \& Mackey, 2001).

Outro aspeto relevante do programa de autotratamento foram os exercícios compensatórios escolhidos para fazer parte da cartilha, todos do princípio de extensão de coluna (deitado em prono, prono extensão, extensão repetida deitado e em pé), pois é a direção que alivia, abole e centraliza a maioria das dores lombares não específicas (Hefford, 2008; Long, Donelson, \& Fung, 2004; Udermann et al., 2004). Cabe ressaltar que os exercícios deviam ser continuados apenas se os pacientes observassem diminuição, abolição ou centralização da dor ou melhora da movimentação da coluna durante ou logo após os exercícios. 
As limitações verificadas no estudo foram: a impossibilidade de alocação aleatória dos idosos nos grupos pela falta de disponibilidade de horário dos mesmos; a amostra ser de conveniência dentro de um grupo de pesquisas, o que pode ter facilitado a aceitação da intervenção proposta; e a alta escolaridade percebida na amostra, o que pode ter favorecido maior entendimento do tratamento proposto, e não permite a extrapolação dos dados para idosos com menor escolaridade. Essas limitações podem ser justificadas pelo fato de o local de coleta ter sido na capital do país, e em uma Universidade. Este é um estudo inicial e justifica-se a realização de um estudo aleatório com amostra maior, mais heterogênea quanto à escolaridade e com período mais longo de acompanhamento para verificação dos efeitos em longo prazo.

\section{CONCLUSÃO}

Os resultados em curto prazo deste programa educacional de autocuidado de coluna demonstram eficácia na diminuição da intensidade da dor, da incapacidade relacionada à dor lombar e na redução dos pontos de dor, em idosos ativos com DL crônica. Destacando a cartilha como uma ferramenta potencialmente útil e capaz de gerar independência no cuidado das dores crônicas da coluna.

\section{Agradecimentos:}

Nada a declarar.

\section{Conflito de Interesses:}

Nada a declarar.

Financiamento:

Nada a declarar.

\section{REFERÊNCIAS}

Airaksinen, O., Brox, J. I., Cedraschi, C., Hildebrandt, J., Klaber-Moffett, J., Kovacs, F., ... Za- noli, G. (2006). Chapter 4 European guidelines for the management of chronic nonspecific low back pain. European Spine Journal, 15(2), S192-S300. http://doi.org/10.1007/s00586006-1072-1

Albaladejo, C., Kovacs, F. M., Royuela, A., del Pino, R., \& Zamora, J. (2010). The efficacy of a short education program and a short physiotherapy program for treating low back pain in primary care: a cluster randomized trial. Spine, 35(5), 483-496.

http://doi.org/10.1097/BRS.0b013e3181b9c9a 7

Arnau, J. M., Vallano, A., Lopez, A., Pellisé, F., Delgado, M. J., \& Prat, N. (2005). A critical review of guidelines for low back pain treatment. $E u-$ ropean Spine Journal, 15(5), 543-553. http://doi.org/10.1007/s00586-005-1027-y

Balagué, F., Mannion, A. F., Pellisé, F., \& Cedraschi, C. (2012). Non-specific low back pain. Lancet, 379(9814),

482-491. http://doi.org/10.1016/S01406736(11)60610-7

Barlow, J., Wright, C., Sheasby, J., Turner, A., \& Hainsworth, J. (2002). Self-management approaches for people with chronic conditions: a review. Patient Education and Counseling, 48(2), 177-187.

Bogduk, N. (2004). Management of chronic low back pain. The Medical Journal of Australia, 180(2), 79-83.

Bombardier, C., Hayden, J., \& Beaton, D. E. (2001). Minimal clinically important difference. Low back pain: outcome measures. The Journal of Rheumatology, 28(2), 431-438.

Brazil, A. V., Ximenes, A. C., Radu, A. S., Fernades, A. R., Appel, C., Maçaneiro, C. H., ... Stump, X. M. G. (2004). Diagnóstico e tratamento das lombalgias e lombociatalgias. Revista Brasileira de Reumatologia, 44(6), 419-425. http://doi.org/10.1590/S048250042004000600005

Clark, D. O. (1996). Age, socioeconomic status, and exercise self-efficacy. The Gerontologist, 36(2), 157-164.

Costa, L. O. P., Maher, C. G., \& Latimer, J. (2007). Self-report outcome measures for low back pain: searching for international cross-cultural adaptations. Spine, 32(9), 1028-1037. http://doi.org/10.1097/01.brs.0000261024.27 926.0f 
Costa, L. O. P., Maher, C. G., Latimer, J., Ferreira, P. H., Pozzi, G. C., \& Ribeiro, R. N. (2007). Psychometric characteristics of the BrazilianPortuguese versions of the Functional Rating Index and the Roland Morris Disability Questionnaire. Spine, 32(17), 1902-1907. http://doi.org/10.1097/BRS.0b013e31811eab3 3

Dagenais, S., Tricco, A. C., \& Haldeman, S. (2010). Synthesis of recommendations for the assessment and management of low back pain from recent clinical practice guidelines. The Spine Journal, 10(6), 514-529. http://doi.org/10.1016/j.spinee.2010.03.032

Damush, T. M., Weinberger, M., Perkins, S. M., Rao, J. K., Tierney, W. M., Qi, R., \& Clark, D. O. (2003). The long-term effects of a selfmanagement program for inner-city primary care patients with acute low back pain. $A r$ chives of Internal Medicine, 163(21), 26322638.

http://doi.org/10.1001/archinte.163.21.2632

Dionne, C. E., Dunn, K. M., \& Croft, P. R. (2006). Does back pain prevalence really decrease with increasing age? A systematic review. Age and Ageing, 35(3), 229-234. http://doi.org/10.1093/ageing/afj055

Engers, A., Jellema, P., Wensing, M., van der Windt, D. a. W. M., Grol, R., \& van Tulder, M. W. (2008). Individual patient education for low back pain. The Cochrane Database of Systematic Reviews, (1), CD004057. http://doi.org/10.1002/14651858.CD004057. pub3

Haas, M., Groupp, E., Muench, J., Kraemer, D., Brummel-Smith, K., Sharma, R., ... Fairweather, A. (2005). Chronic disease selfmanagement program for low back pain in the elderly. Journal of Manipulative and Physiological Therapeutics, 28(4), 228-237. http://doi.org/10.1016/j.jmpt.2005.03.010

Harrison, D. D., Harrison, S. O., Croft, A. C., Harrison, D. E., \& Troyanovich, S. J. (1999). Sitting biomechanics part I: review of the literature. Journal of Manipulative and Physiological Therapeutics, 22(9), 594-609.

Hefford, C. (2008). McKenzie classification of mechanical spinal pain: profile of syndromes and directions of preference. Manual Therapy, 13(1), 75-81. http://doi.org/10.1016/j.math.2006.08.005
Heymans, M. W., van Tulder, M. W., Esmail, R., Bombardier, C., \& Koes, B. W. (2004). Back schools for non-specific low-back pain. The Cochrane Database of Systematic Reviews, (4), CD000261. http://doi.org/10.1002/14651858.CD000261. pub2

Hicks, G. E., Gaines, J. M., Shardell, M., \& Simonsick, E. M. (2008). Associations of back and leg pain with health status and functional capacity of older adults: findings from the retirement community back pain study. Arthritis and Rheumatism, 59(9), 1306-1313. http://doi.org/10.1002/art.24006

Jensen, M. P., Karoly, P., \& Braver, S. (1986). The measurement of clinical pain intensity: a comparison of six methods. Pain, 27(1), 117-126. http://doi.org/10.1016/0304-3959(86)902289

Kovacs, F., Abraira, V., Santos, S., Díaz, E., Gestoso, M., Muriel, A., ... Spanish Back Pain Research Network. (2007). A comparison of two short education programs for improving low back pain-related disability in the elderly: a cluster randomized controlled trial. Spine, 32(10), 1053-1059.

http://doi.org/10.1097/01.brs.0000261556.84 266.0f

Leboeuf-Yde, C., Nielsen, J., Kyvik, K. O., Fejer, R., \& Hartvigsen, J. (2009). Pain in the lumbar, thoracic or cervical regions: do age and gender matter? A population-based study of 34,902 Danish twins 20-71 years of age. BMC Musculoskeletal Disorders, 10, 39. http://doi.org/10.1186/1471-2474-10-39

Lima, M. G., Barros, M. B. de A., César, C. L. G., Goldbaum, M., Carandina, L., \& Ciconelli, R. M. (2009). Impact of chronic disease on quality of life among the elderly in the state of São Paulo, Brazil: a population-based study. Revista Panamericana De Salud Pública, 25(4), 314321.

Long, A., Donelson, R., \& Fung, T. (2004). Does it matter which exercise? A randomized control trial of exercise for low back pain. Spine, 29(23), 2593-2602.

Matsudo, S., Araújo, T., Marsudo, V., Andrade, D., Andrade, E., Oliveira, luis C., \& Braggion, G. (2001). Questinário internacional de atividade física(IPAQ): estudo de validade e reprodutibilidade no Brasil. Revista Brasileira de Atividade Física \& Saúde, 6(2), 5-18. 
May, S. (2010). Self-management of chronic low back pain and osteoarthritis. Nature Reviews. Rheumatology, 6(4), 199-209. http://doi.org/10.1038/nrrheum.2010.26

McKenzie, R. (2007). Trate você mesmo sua coluna. (J. L. F. Barros, Trad.) (2a Edição Traduzida). Belo Horizonte: TTMT.

Meng, K., Seekatz, B., Roband, H., Worringen, U., Vogel, H., \& Faller, H. (2011). Intermediate and long-term effects of a standardized back school for inpatient orthopedic rehabilitation on illness knowledge and self-management behaviors: a randomized controlled trial. The Clinical Journal of Pain, 27(3), 248-257. http://doi.org/10.1097/AJP.0b013e3181ffbfaf

Nusbaum, L., Natour, J., Ferraz, M. B., \& Goldenberg, J. (2001). Translation, adaptation and validation of the Roland-Morris questionnaire-Brazil Roland-Morris. Brazilian Journal of Medical and Biological Research, 34(2), 203210.

Oliveira, V. C., Ferreira, P. H., Maher, C. G., Pinto, R. Z., Refshauge, K. M., \& Ferreira, M. L. (2012). Effectiveness of self-management of low back pain: systematic review with metaanalysis. Arthritis Care \& Research, 64(11), 1739-1748. http://doi.org/10.1002/acr.21737

Pynt, J., Higgs, J., \& Mackey, M. (2001). Seeking the optimal posture of the seated lumbar spine. Physiotherapy Theory and Practice, 17(1), 5-
21.

http://doi.org/10.1080/09593980151143228

Udermann, B. E., Spratt, K. F., Donelson, R. G., Mayer, J., Graves, J. E., \& Tillotson, J. (2004). Can a patient educational book change behavior and reduce pain in chronic low back pain patients? The Spine Journal, 4(4), 425-435. http://doi.org/10.1016/j.spinee.2004.01.016

Van den Bussche, H., Koller, D., Kolonko, T., Hansen, H., Wegscheider, K., Glaeske, G., ... Schön, G. (2011). Which chronic diseases and disease combinations are specific to multimorbidity in the elderly? Results of a claims data based cross-sectional study in Germany. BMC Public Health, 11, 101. http://doi.org/10.1186/1471-2458-11-101

Van Middelkoop, M., Rubinstein, S. M., Verhagen, A. P., Ostelo, R. W., Koes, B. W., \& van Tulder, M. W. (2010). Exercise therapy for chronic nonspecific low-back pain. Best Practice \& Research. Clinical Rheumatology, 24(2), 193-204.

http://doi.org/10.1016/j.berh.2010.01.002

Vita, A. J., Terry, R. B., Hubert, H. B., \& Fries, J. F. (1998). Aging, health risks, and cumulative disability. The New England Journal of Medicine, 338(15), 1035-1041. http://doi.org/10.1056/NEJM1998040933815 06

Todo o conteúdo da revista Motricidade está licenciado sob a Creative Commons, exceto quando especificado em contrário e nos conteúdos retirados de outras fontes bibliográficas. 